\title{
Exploring the truthiness in home sleep test (HST) reports
}

\author{
Douglas E. Moul $^{1}$ (D)
}

Received: 14 December 2014 /Revised: 11 May 2015 / Accepted: 2 June 2015 / Published online: 12 June 2015

(C) Springer-Verlag Berlin Heidelberg 2015

Recently, our sleep center reengaged the question of what standard form of wording to put in the conclusions section of home sleep test (HST) reports about the severity of obstructive sleep apnea (OSA). One staff member opined that it should merely say that the study did or did not confirm an OSA diagnosis, irrespective of AHI severity, but feared that bringing up this wording issue would open up a "can of worms." One technologist made wording suggestions which were not accepted. The complexities across cases make any standard wording liable to charges of "truthiness" or "shallow sophistry." The "can of worms" characterization expressed an honest anxiety about the political and philosophical complexities. In any center processing larger number of HSTs, what wording(s) can balance the dual goals of being efficient while fostering good clinical care?

This "can of worms" indeed will not just go away. Resolving its political aspects will depend on the particular sleep center. However, a deeper analysis would suggest that this "can of worms" has at least two main lines of conceptual complexity for individual HST reports, irrespective of local politics. The first is about what theory(ies) of truth (i.e., validity) to utilize when working through to a study's interpretation. While there are newer, minor variants for theories of truth, the main enduring ones are the Correspondence Theory of Truth (CORRESTT) and the Coherent Theory of Truth (COHERTT) [1].

The CORRESTT is a notion that a statement is true if it corresponds to the state of affairs "in reality." We use this

Douglas E. Moul

mould@ccf.org

1 Sleep Disorders Center, Cleveland Clinic, FA20/9500 Euclid Avenue, Cleveland, OH 44195, USA general approach in conforming to official diagnostic criteria, even when its strict observance is an oversimplification of how science works [2]. According to diagnostic criteria, if particular clinical observations are present, and fit the criteria, then the patient has the medical condition. For example, a respiratory event is scored as a hypopnea because one follows the applicable scoring criteria for hypopneas, and not otherwise. The extreme position for the CORRESTT is positivism, a position supporting summative conclusions only if positive observable evidence according to standardized rules of observation is present. But under such an extreme positivistic standard, the apnea-hypopnea index (AHI) estimated from an HST cannot support any OSA diagnosis, since the rules for the HST are not those for the polysomnogram (PSG). This extreme kind of positivism is long out of date, although sometimes still proffered because it emphasizes observational reliability. While using standardized scoring criteria is highly important for inter-rater reliability, reliability is not the only aspect of validity.

By contrast, the COHERTT states that a statement can be considered true when it is in general coherence with the real situation. This kind of truth assessment is not based on abiding strictly by a fixed set of rules but is more applicable in ambiguous fact situations where a reasoned judgment is required about where the truth lies. The current controversy about whether those patients with $\mathrm{AHI}<5$ according to the Centers for Medicare and Medicaid Services (CMS) $4 \%$ rule, but with an $\mathrm{AHI}>5$ according to the American Academy of Sleep Medicine (AASM) $3 \%$ rule, illustrates this point. Do such CMS $4 \%$-negative patients "not have" OSA? Applying CMS rules strictly by the CORRESTT, no; but this conclusion is absurd. Of course that patient has OSA, especially if the AHI is greater than 30 per AASM criteria! It is simply false (compellingly by the COHERTT) that that patient does not have OSA. While we are waiting for the $4 \%$ rule to be 
changed, the wise scorer will mediate the case-specific conflicts between CORRESTT and COHERTT. The COHERTT approach allows some truth assessment based on the global fact situation beyond fixed rules. Conclusions derived from CORRESTT-based rules that when applied strictly do not make good "COHERTT" sense are indeed untruthful. We all know that even nominally reliable rules of judgment can be inappropriate in some situations.

The second line of complexity is that medical report writing also involves ethical considerations. Since the early 20th century, medical practice and opinion have placed high ethical value that medical reasoning be science and evidence based. Sometimes, this perspective has been over-valued to the point where no treatment or diagnostic process can be "justified" without several randomized clinical trials conclusively ruleproving its benefits. Practices outside "practice parameters" can take on a caste of being immoral under this severe epistemological regime. One problem with this level of positivism is that medicine is not truthfully a science but a practical activity like engineering. Clinicians work out problems for patients individually. Clinicians are not mainly concerned with discovering generalizabilities, as science is. Medical science is important, but clinicians take care of unique individual patients. Within the scope of interpreting PSGs and HSTs, this means that making treatment recommendations on reports is mainly an ethical task of conditionally addressing the individual patient's complexities and the referring clinician's level of knowledge about sleep medicine. While there are operational advantages to using standard, "generalizable" wording on HST reports, these standard wordings are appropriate only when they adequately address the patient's medical needs.

My ethical view, then, about the validity of judgments concerning HST ratings of severity is that the application of which theory(ies) of truth to apply is dependent on the AHI range and other data from the clinical chart and the HST readout. For low AHIs, one is obliged to apply the CORRESTT especially more rigorously when little or no supine sleep is observed. For example, I had one non-diagnostic off-supine HST that when on subsequent supine PSG was found to have an AHI of 102 ! So strictly observing the scoring and interpretive rules is indeed sometimes more like an ethical imperative, especially if a study's low AHI raises the test insensitivity of HST as a greater concern. When $4 \%$ CORRESTT-style rule application seriously underrepresents the seriousness where application of $3 \%$ rules would not, liberal CORHERTT scoring of events is then an ethical priority for the patient's medical interest. Additionally, for high AHIs on HSTs where the OSA diagnosis is obvious, then the application of the COHERTT should be applied more actively in choosing the report wording, since a study with high AHI and substantial desaturation calls for assertive language on the HST report. So I do not believe that the choice of which theory of truth to apply to HST AHI estimates can be completely dissociated, ethically or epistemologically, from the individual patient's clinical context. In my opinion, the choice of what specific standard form of words to put in the report is ethically superseded by case-specific decisions about which theory of truth to apply, as conditioned by the AHI, desaturations, and other ethical aspects of the case. This conditionality certainly depreciates the imposition of an unconditional wording that would be applied to intermediate cases as well.

An HST report's wording is the interpreter's validity meta-problem. One should choose each report's wording accurately, while providing sufficiently reflective, situation-specific guidance for the patient and ordering physician's needs. This meta-problem undermines strict inter-rater reliability, since interpretive judgments are necessarily made by a situated interpreter who makes choices about the applicable theories of truth to apply in individual cases. This was previously noted in a study of physicians' interpretations of overnight pulse oximetry monitoring [3]. The HST report is fundamentally artisanal. This artisanal method of ethical judgment is termed "casuistry," denoting that such judgments are naturally so conditional that a fixed rule cannot be uniformly applied to all cases [4]. While likely less "reliable" in the interrater reliability sense, such situated judgments will nonetheless remain subject to debate and comparison [3], since different cases have relations between them of verisimilitude (i.e., a kind of ethical truth) that do guide professional consensus about interpretations' appropriateness in similar situations. As much practice parameters may serve as one theory of truth; HST reports still concern individuals' unique needs. Clinical judgment is done not only mindful of some norming for scientific generalizability and procedural standardization but also artistically in relation to the clinical situation. In the language of utilitarianism, this interpretive attitude can be said to favor artful, situation-conditional utilitarianism marginally over strict rule utilitarianism: The needs of the one can sometimes outweigh the rules, or the wordings, for the many. Otherwise, it would be enough to decide what the consensus rules and wordings should be-in the spirit of a Tarskian Theory of Truth-and just let obedient technologists, or computer algorithms, apply them uniformly on all HST reports. Absolutely uniform interpretive wording rules will reduce overall work burden in a busy laboratory but pose the danger that absolutely standard wording would medically deskill the interpreter by enforcing interpretive monotony.

Conflict of interest The author declares that he has no competing interests.

Disclaimer This essay reflects my views, and not necessarily those of my employer or colleagues. I thank the reviewers and editor for their help. 


\section{References}

1. Schmitt FF (2004) Theories of truth. Blackwell Pub, Malden

2. Quine WV (1960) Word and object. Technology Press of the Massachusetts Institute of Technology, Cambridge
3. Ramsey R, Mehra R, Strohl KP (2007) Variations in physician interpretation of overnight pulse oximetry monitoring. Chest 132(3):852-9

4. Beauchamp TL, Childress JF (2009) Principles of biomedical ethics, 6th edn. Oxford University Press, New York 